\title{
Anaesthetic Management of Hemiparesis Patient with Severe Thoracolumbar Scoliosis for Ortho- paedic Surgery by Taylor 's Approach of Lumbar Subarachnoid Block in the era of COVID 19 Pandemic
}

P Veena ${ }^{*}$; Mohankumar ${ }^{2}$; Geethakumari P2; Arun $R^{3}$

${ }^{1}$ Assistant professor, Department of Anaesthesiology, SUT Academy of Medical Sciences, Thiruvananthapuram, kerala, India. ${ }^{2}$ Professor and Head of the Department of Anaesthesiology, SUT Academy of Medical Sciences, Thiruvananthapuram, kerala, India. ${ }^{3}$ Assistant Professor, Department of Anaesthesiology, SUT Academy of Medical Sciences, Thiruvananthapuram, kerala, India. ${ }^{4}$ Senior Resident, Department of Anaesthesiology, SUT Academy of Medical Sciences, Thiruvananthapuram, kerala, India.

\section{*Corresponding Author(s): P Veena}

Assistant professor, Department of Anaesthesiology, SUT Academy of Medical Sciences, Thiruvananthapuram, kerala, India.

Email: veenap743@gmail.com

Received: May 24, 2021

Accepted: Jun 17, 2021

Published Online: Mar 08, 2021

Journal: Annals of Anesthesia and Pain Medicine

Publisher: MedDocs Publishers LLC

Online edition: http://meddocsonline.org/

Copyright: (C) Veena P (2021). This Article is

distributed under the terms of Creative Commons

Attribution 4.0 International License

Keywords: Taylor's approach; Lumbar subarachnoid block; Paramedian; Proximal femur nailing; thoracolumbar scoliosis; Hemiparesis.

\section{Abstract}

Background: Subarachnoid block in patients with scoliosis always present a unique challenge to the anesthesiologist owing to the deformity of spine. Difficulty in performing neuraxial anaesthesia may result in neural injury, spinal haematoma, post-dural puncture headache In addition, it may decrease procedure efficiency and increase patient discomfort and dissatisfaction. The most commonly practiced technique is the midline approach. This approach is technically difficult in the geriatric patients because of degenerative changes in the spine. Calcification of supraspinous and interspinous ligaments in the geriatric age group makes midline approach difficult. Paramedian approach and its modification Taylor's approach is not routinely practiced and is definitely a game changer when midline approach of lumbar subarachnoid block has failed or is not possible due to anatomical variations like ankylosing spondylitis, thoracolumbar kyphoscoliosis.

We report a case of unilateral hemiparesis patient with severe thoracolumbar scoliosis anaesthetically managed by Taylor's approach of lumbar Subarachnoid block for Proximal Femur Nailing (PFN) of intertrochanteric fracture thus avoiding general anaesthesia and associated aerosol generation in the era of COVID19 pandemic.

Cite this article: Veena P, Mohankumar, Geethakumari P, Arun R. Anaesthetic Management of Hemiparesis Patient with Severe Thoracolumbar Scoliosis for Orthopaedic Surgery by Taylor 's Approach of Lumbar Subarachnoid Block in the era of COVID 19 Pandemic. Ann Anesth Pain Med. 2021; 4(1): 1021. 


\section{Introduction}

Subarachnoid blockade is widely used due to its procedural simplicity, low cost and better physiological benefits and thus reduced complications than that of general anaesthesia. Subarachnoid block can be safely administered via Taylor's approach for anticipated as well as unanticipated difficult spine cases when general anaesthesia has to be avoided as far as possible to prevent aerosol generation during era of COVID19 Pandemic. Severe thoracolumbar scoliosis with hemiparesis following a cerebrovascular accident in a geriatric age group holds array of challenges for both general anaesthesia and regional anaesthesia. Considering the prevailing COVID19 Pandemic and thoracolumbar scoliosis with hemiparesis its always safe to proceed with regional anaesthesia as far as possible. Subarachnoid block is preferred by many anesthesiologists in view of cost effectiveness, low risk of cognitive dysfunction, thrombo-embolic events, post-operative respiratory morbidity, renal failure and prolonged post-operative hospital stay.

\section{Case report}

We report a case of 80 year old female patient anaesthetically managed with Taylor's approach of lumbar subarachnoid block for right Proximal Femur Nailing (PFN). Known case of diabetes, hypertension, History of cerebrovascular accident 1 year back with residual hemiparesis of right side on dual antiplatelet drugs of which clopidogrel was stopped 5 days prior to surgery. No history of other major illness and surgery. On examination the patient had adequate mouth opening and airway examination revealed an anticipated difficult airway with partially edentulous, Mallampatti class 3, short neck and with normal temperomandibular joint movement. Intravenous access as well as regional anaesthesia access difficultly was anticipated. Examination of spine revealed severe thoracolumbar scoliosis. Airentry was equal on both sides. Blood investigations after stopping clopidogrel were normal. Chest Xray revealed cardiomegaly. Neurological assessment revealed right side residual hemiparesis. Central neuraxial block is controversial with dual antiplatets in these patients and poses an anesthetic challenge, in view of difficulties in palpating anatomical landmarks, performing dura puncture, and difficulty in predicting the extent of block. We followed the updated universal gold standard ASRA (American society of Regional Anaesthesiology) guidelines regarding antiplatet medication.

\section{Anaesthetic management}

After arranging adequate blood products, a detailed written informed high risk consent was obtained for the planned anaesthesia procedure along with a backup plan for general anaesthesia in view of anticipated difficult regional anaesthesia access. Sedative premedications were avoided, Patient was reassured to ally anxiety and was given T. Pantoprazole 40mg, along with patient 's own medication, N.P.O was maintained.

After preparation of OT, checking anaesthesia machine with emergency resuscitation drugs, intravenous fluids, infusion pump, difficult regional anaesthesia equipments and equipment backup for general anaesthesia patient was shifted to OT.

On receiving inside OT - Monitors for ECG, Non-invasive Blood pressure, pulse oximetry, temperature probe were connected. Two wide bore cannulae were established under strict asepsis under local anaesthesia and was preloaded judiciously with balanced salt solution.
Under strict asepsis, Inspite of adequate interspinous space, negotitating a 23G Quincke 's spinal needle was difficult and needle was hitting bone in midline as well as in paramedian approach. Hence considering the prevailing covid pandemic and to avoid general anaesthesia for the patient we reassured the patient and reverted the patient to sitting position for a modified paramedian approach "Taylor's Approach" of subarachnoid blockade. Taylor's approach attempted under strict asepsis with 23G Quincke spinal needle. Posterior Superior Iliac Spine (PSIS) palpated, $1 \mathrm{~cm}$ medial and $1 \mathrm{~cm}$ caudal to PSIS needle inserted and directed towards cephalo-medial direction. After advancing needle bony obstruction was felt. Walking over the bone "Loss of Resistance" was elicited and stylet removed. Position of needle tip in subarachnoid space confirmed by clear and free backflow of cerebrospinal fluid. $2.8 \mathrm{ml}$ of $0.5 \%$ heavy bupivacaine with 30 microgram buprenorphine was given. Block adequacy was confirmed in supine position. vitals stable and intraoperatively one pint whole blood was transfused. Surgery completed uneventful. postop period was uneventful .

\section{Discussion}

Geriatric patients undergoing major surgery have a significantly higher incidence of morbidity and morality compared with younger age group because of their reduced cardio respiratory reserve and concomitant diseases .Spinal anaesthesia is widely used in orthopaedic surgery. Scoliosis (lateral spinal curvature) and in majority, it is usually an idiopathic disorder. Secondary kyphoscoliosis occurs as a result of various neuromuscular, vertebral, or connective tissue disorders. The level of derangements in cardiac and pulmonary function of kyphoscoliosis patient is related to the amount of Cobb's angle in thoracolumbar X-ray. Cardiopulmonary function starts decreasing if this angle is larger than $40^{\circ}$ and it becomes very significant with angle $>100$. In our patient, post cerebrovascular accident induced hemiplegia 1 year back was the cause of progressive thoracolumbar scoliosis, but he had no significant respiratory or cardiovascular instability. Spinal anesthesia in this type of patient is technically challenging to the anesthesiologist. Due to angulation and rotation of vertebral body, epidural space is deviated toward the convexity of angulation. One method of administering spinal or epidural block is by directing the needle toward the convexity of the curve with significant angulation of the needle. Because of the distortion of the spinous processes in patients with scoliosis, the interspinous space is not felt. Modified paramedian approach "Taylor 's approach" is definitely a game changer in such challenging spine deformity cases.

\section{Conclusion}

Your hands will do only what the mind thinks of so be updated in knowledge as well as in skill regarding how to tackle such challenging regional anaesthesia cases in prevailing COVID19 pandemic condition. Taylor 's approach is the best modified paramedian approach in difficult spine access for lumbar subarachnoid block though only a few is aware of such an approach.

\section{Acknowledgement}

Department of Anaesthesiology, Department of orthopaedics, OT \& RICU staffs SUT Academy of Medical Sciences, Thiruvananthapuram, kerala, India. 


\section{References}

1. Brull R, Macfarlane AJ, Chan VW, Miller RD, Cohen NH, et al. Young Spinal, Epidural, and Caudal Anesthesia Miller's Anesthesia Eighth Edition Elsevier Philadelphia. 20151692

2. Center NCG. The Management of Hip Fracturein Adult. Regional vs General Anaesthesia. London: Royal College of Physician. 2011.

3. Asra Guidelines Anticoagulation Pdf (helpforchildren. info).

4. Chin KJ, Macfarlane AJR, Chan V, Brull R. The use of ultrasound to facilitate spinal anesthesia in a patient with previous lumbar laminectomy and fusion: A case report J Clin Ultrasound. 2009378482510.1002/jcu.20588
5. https://www.academia.edu/47848195/The_Role_of_ Perioperative_Physicians...

6. Taylor\%E2\%80\%99s-Approach (ijmsir.com)

7. (PDF) Taylor's Approach/Modified paramedian approach a game changer in unanticipated difficult spine during the era of Covid pandemic - A Case Report (researchgate.net)

8. Guidelines for Neuraxial Anesthesia and Anticoagulation (ihc.com). 